Health plan administrators and clinicians have pre-existing ideas of research that necessitate ongoing education and dialogue to address concerns. Using a framework of change native to the health system promotes navigation of potentially disruptive change. Given rapid changes within health systems, the research team must be aware of competing contextual factors. Finally, a true health system/research partnership when staffing the intervention facilitates sustainability of intervention related services. Conclusions: A pragmatic trial approach, although better suited for embedded research in the healthcare system, presents challenges not typically encountered in standard explanatory/ efficacy trials. Lessons learned by this team that accommodate both healthcare operations and research can promote embedded research in other health settings.

Keywords: Pragmatic clinical trial; Chronic pain

doi:10.3121/cmr.2014.1250.a3-4

\section{A3-5: \\ Research-Operations Partnerships to Improve the Quality and Affordability of Care}

Michael Gould ${ }^{1}$; Huong Nguyen ${ }^{1}$; Adam Sharp ${ }^{1}$; Erin Hahn'; Tania Tang ${ }^{1}$; Brian Mittman ${ }^{1}$; Steven Jacobsen ${ }^{1}$; Michael Kanter ${ }^{1}$

\section{${ }^{1}$ Kaiser Permanente Southern California}

Background/Aims: A learning health care organization requires visionary leadership to achieve the triple aim of better health, better care, and improved affordability. Effective research-operations partnerships (ROPs) can facilitate improvement efforts. Although ROPs are not novel, the process of establishing sustainable collaborations and overcoming challenges to achieve shared objectives is not well described. Methods: In 2012, the executive leadership of Kaiser Permanente Southern California (KPSC) made a multi-million dollar investment by creating the Care Improvement Research Team (CIRT) within the Department of Research and Evaluation. Members of this team developed and refined ideas about ROPs by reviewing published literature, conducting semi-structured interviews with key operational leaders, consulting with experts, and sponsoring an all-day retreat on this theme. Results: Key elements of the initiative include: (1) recruitment of health care researchers with complementary clinical and methodological experience, supported by an expert consultant in implementation science; (2) selection of research questions that are clearly aligned with organizational priorities; (3) development of strong, sustainable relationships with key stakeholders across multiple levels of the organization, through outreach and embedding of researchers in operational work groups; (4) dedication of internal funds to cover effort of research support staff; (5) creation of a strategic plan to define roles, responsibilities, and goals that allow for measurement of the program's success. Key challenges to overcome include: (1) limited availability of external funds to support research that addresses operational imperatives; (2) mismatched timelines and incentives; (3) legal constraints that hamper data sharing between research and operations; and (4) balancing research rigor with operational relevance. Conclusions: Prioritizing competing initiatives, identifying opportunities for early success, and aligning operational priorities with research opportunities will require continued effort. Given the austere external funding environment, novel ways to support delivery system science are needed. The creation of the CIRT at KPSC represents a strategic investment from visionary leaders who embody the credo to "be the best at getting better" and who recognize that the development of strong and sustainable ROPs will be invaluable if we are to achieve the triple aim.

Keywords: Partnerships; Care improvement

doi:10.3121/cmr.2014.1250.a3-5

PS1-20:

Understanding Patient Barriers and Preferences to Completing Advance Directives (AD) in the Primary Care Setting

J.B. Jones ${ }^{1}$; Janet Tomcavage ${ }^{1}$; Dorothy Fisher ${ }^{2}$; Ryan Van Loan ${ }^{1}$; Virginia Lerch $^{1}$; Thomas Graf ${ }^{1}$

\section{Geisinger Health System; ${ }^{2} \mathrm{New}$ West Health Services}

Background/Aims: Advance Care Planning (ACP) is a complex process that allows individuals to contemplate and document end of life decisions using tools such as an Advance Directive (AD). The proportion of patients who have an $\mathrm{AD}$ on file remains low both nationally and at Geisinger. To date, little research has focused on healthy populations' attitudes towards ACP and AD completion. We describe the design and implementation of a web-based application to collect patients' preferences for and barriers to $\mathrm{AD}$ completion in a large, non-diseased primary care population. Methods: We developed a simple web application and questionnaire (denoted "eACP") designed to educate patients about completing an AD. The eACP application was automatically presented on a touchscreen computer to all patients aged 50-64 who were seen in one of 5 Geisinger Clinic locations for a routine appointment. The questionnaire introduced ACP as a part of good healthcare and asked patients if they were interested in learning more. Patients who chose not to learn more indicated why they declined. Patients who elected to learn more selected topics of $\mathrm{AD}$ completion for which they would like more information and indicated how they wished to review the information. Results: A total of 2169 patients completed the questionnaire using the eACP application in 5 practice sites between $07 / 31 / 13$ and 10/30/13. Nearly $40 \%$ $(852 / 2169)$ of patients were interested in learning more while $49.8 \%$ (1080/2169) were not. The primary reasons for declining to learn more included lack of time, a preference for leaving the choice to others, or prior $\mathrm{AD}$ completion. Among the patients who elected to learn more, the most common topics of interest were related to the process of completing an $\mathrm{AD}$ (e.g., what goes into an $\mathrm{AD}$ and how/when to complete it). Patients had a strong preference for printed materials $(70 \%)$ versus using a website $(30 \%)$ or talking to a healthcare professional $(<10 \%)$. Conclusions: Our findings suggest that patients desire more education on ADs but prefer to receive it in a paper format versus online or via a discussion with their provider. Strategies for increasing AD completion in practice should account for these patient preferences.

Keywords: Patient-reported data; Advance care planning

doi:10.3121/cmr.2014.1250.ps1-20

PS1-21:

Development and Dissemination of Quality Review Tools for Data Management and Analysis

Sophia Newcomer ${ }^{1}$; Liza Reifler ${ }^{1}$; Jennifer Ellis ${ }^{1}$

${ }^{1}$ Kaiser Permanente Colorado

Background/Aims: Within embedded HMO research groups, analytic teams are heavily involved with many key stages of research studies. Individual analytic team members use a variety of processes for ensuring work quality. Identification and standardization of analytic quality review best practices may lead to greater efficiencies and fewer errors. The objective of this quality improvement project was to develop and implement tools to enhance the quality and consistency of analytic work within a HMO-based research group. Methods: We used a multi-modal approach to develop and assess analytic quality review tools. We first conducted four in-depth interviews with principal investigators to collect experiences and suggestions for improving analytic processes. Using an online survey, we collected best practices from analytic team members. We also interviewed analytic leads with $6 \mathrm{HMO}$ and academic partner research groups on their best practices. We integrated all information and developed analytic quality review best practice documentation and tools. We tested and revised the tools, estimated time requirements, and provided basic training to our analytic team. Nine months later, we conducted an anonymous online survey to gauge uptake of these tools and to collect initial feedback from early adopters. Results: Four analytic quality review tools were developed to help formalize best practices for cohort-building/data pulls, data preparation (code review), analytic dataset preparation and documentation, and manuscript methods and results review. In initial assessments, implementing the code review was the most resource-intensive, taking upwards of 14 hours. Nine months after introducing these tools, the most commonly reported challenges to using the tools included lack of time/funding, using other processes to ensure work quality, and lack of knowledge on when or how to use the tools. Early adopters gave qualitative feedback that the tools helped structure analytic processes and encouraged documentation of analytic decisions. Conclusions: Identification and standardization of best practices may have the potential to improve analytic work processes for HMO research groups. Future efforts should focus on quality review tool revisions, policies for tool use, data collection 